\title{
Health-Promoting Lifestyles of English-Speaking and Spanish- Speaking Mexican-American Migrant Farm Workers
}

\author{
Madeleine J. Kerr, M.S., R.N. and \\ Deborah A. Ritchey, M.S., R.N.
}

\begin{abstract}
This study was conducted with 62 Mexican-American migrant farm workers at four different sites in northern Illinois. An established English and a newly developed pilot Spanish version of the health-promoting lifestyle profile was used. The concept of health-promoting lifestyle appeared to be culturally relevant to study participants. English-speaking migrant workers scored significantly lower than Spanishspeaking workers on the dimensions of self-actualization, exercise, and stress management. Patterns of scores among both groups were highest in self-actualization and interpersonal support, and lowest in health responsibility and exercise. Further research in health-promoting behaviors with all cultural groups and socioeconomic levels of society will contribute to achievement of the World Health Organization's goal, health for all by the year 2000 .
\end{abstract}

Madeleine J. Kerr, M.S., R.N. is a Doctoral Student, the University of Michigan School of Nursing, Ann Arbor, MI

Deborah A. Ritchey. M.S., R.N. is an Outreach Nurse, Crusader Health Care for the Homeless Program, Rockford, Illinois

Supported by a grant from the Northern Illinois University School of Nursing. DeKalb, Illinois.

Address correspondence to Madeleine J. Kerr, M.S., R.N., University of Michigan School of Nursing, 400 North Ingalls Building, Ann Arbor, MI 48109-0482.
Is health promotion for everyone, or merely for those who are healthy, well educated, and economically stable? Health promotion has taken root in white, middleclass America in both professional and popular realms. Corporate, hospital, and fitness center programs have emerged, offering a kaleidoscope of activities to the public. Articles in lay health journals, cable television programs, and wellness tools such as relaxation tapes and home rowing machines are available to assist consumers in enhancing their own health. Such resources may not be accessible to all who could benefit from them, however. How can health, defined as high-level wellness, be achieved by diverse cultural, ethnic, and socioeconomic groups? If all segments of society are to have the opportunity to enjoy wellness, it is necessary to conduct health-promotion research with diverse populations and to implement culturally appropriate programs that will enable them to enhance their health.

One American subculture is the Mexican-American migrant farm worker. Although the health problems inherent in migrant farm work have been investigated (Smith, 1986; Wilk, 1986), no research has been done on the health practices of these workers. Knowledge of the health-promoting lifestyle patterns of this population would be very useful in targeting areas to be addressed and identifying the practices that exist to support changes.

Health-promoting behaviors are distinct from prevention behaviors (Pender, 1987). Stated simply, the former are approach behaviors with movement toward health, and the latter are avoidance behaviors with movement away from disease. The two are complementary components of a healthy lifestyle. The study of the health- 
promoting component has recently been facilitated by the development of the health-promoting lifestyle profile (HPLP) (Walker, Sechrist, \& Pender, 1987). The health-promoting lifestyle is defined as "a multidimensional pattern of self-initiated actions and perceptions that serve to maintain or enhance the level of wellness, self-actualization, and fulfillment of the individual" (p. 77).

The purpose of the present study was to explore the feasibility of studying the health-promoting behaviors of both English-speaking and Spanish-speaking MexicanAmerican migrant farm workers using the HPLP. Specific objectives were to assess the cultural relevance of the concept of health-promoting lifestyle among these workers, to describe their practices, and to identify differences in characteristics and health-promoting behaviors between the two groups of workers. Knowledge of these lifestyle patterns will assist community health nurses to build on client strengths to facilitate a higher level of health.

\section{LITERATURE REVIEW}

A review of the literature showed health-promoting lifestyle patterns of migrant farm workers to be a new area of study. The World Health Organization (1982), in pursuit of its goal of health for all by the year 2000 , confirmed the need for research on health-promoting behavior. For example, while much is known about health-damaging behavior such as smoking, much less is known about patterns that promote health.

Several studies have investigated the perception of health of Hispanics by asking them to rate their own health as excellent, good, fair, or poor. Two studies of Mexican-Americans by the Human Population Laboratory found the perceived health of these individuals to be lower than that of Anglos (Roberts \& Lee, 1980). Similarly, a 1978 survey noted that migrant farm workers in Wisconsin perceived their health to be poorer relative to the perceptions of other groups (Slesinger, 1988).

Health-damaging and preventive health behaviors of Hispanics also were explored. The level of use of preventive medical care, including dental care, vision care, and general physical examinations, among Hispanic migrant farm workers in Wisconsin was low compared to other groups in the United States (Slesinger, 1981). The San Antonio heart study reported that Mexican-Americans are significantly heavier relative to their height than Anglos (Stern, Pugh, Gaskill, \& Hazuda, 1982). In addition, low-income Mexican-Americans are less likely than Anglos to engage in two behaviors that prevent obesity, avoiding sugar and dieting. In a study on ethnicity and lifestyle health risk, Hispanic background was associated with not smoking (Gottlieb \& Green, 1987). In addition, gender differences existed with men more likely to exercise and women more likely to abstain from alcohol. No studies were found focusing on health-promoting lifestyle practices of Hispanics.

Acculturation was proposed as an important factor contributing to cultural norms and health behaviors. Significant associations were found between acculturation as measured by language use and health behaviors such as use of oral contraceptives, fatalism regarding health, and attitudes toward folk healers (Deyo, Diehl, Hazuda, \& Stern, 1985). In contrast, cultural assimilation made no difference in preventive health behaviors practiced by elderly Hispanic women (Marks et al., 1987). Finally, the extent of acculturation affected the clinical validity of a Spanish version of a questionnaire that had a high level of reliability (Deyo, 1984). The literature indicates that acculturation may have an influence on health behaviors practiced and on the validity of health questionnaires in the Spanish language, and therefore it can produce meaningful subgroups in populations that may otherwise appear to be homogeneous. For this reason, an acculturation scale was used in the present study to determine language preference of the migrant workers.

\section{METHODS}

Health-promoting lifestyle behaviors were measured by the HPLP (Walker, Sechrist, \& Pender, 1987). This 48item, English-language instrument measures health-promoting behaviors in six dimensions: nutrition, exercise, health responsibility, stress management, interpersonal support, and self-actualization. The lack of available instrumentation in Spanish formed a barrier to exploring health patterns of Spanish-speaking individuals among the Mexican-American migrant farm workers; therefore, with consultation from the authors of the HPLP, the first step was to translate the tool into the Spanish dialect typical of Hispanics of Texas-Mexican origin. A back-translation technique (Berkanovic, 1980) was used to ensure an accurate denotation and connotation of the items in the instrument. The HPLP was translated into Spanish by a bilingual graduate nursing student, and the Spanish version was then back-translated into English by a Mexican-American consultant who had not seen the original English version. The back-translated copy was compared to the original English HPLP by the investigator and one of its authors to identify discrepancies. Three translators were then consulted to correct the discrepancies and revise the pilot Spanish version of the HPLP.

A questionnaire consisting of 12 multiple-choice and completion questions in English and Spanish versions 
was used to collect demographic data about age, sex, marital status, education, years in farm work, perceived health status, and use of folk medicine. The questionnaire also included four brief questions on English language use as described by Deyo et al. (1985), which were used to calculate an index of acculturation. The purposes of the acculturation index were to describe the heterogeneous sample and to explore its usefulness in selecting Spanish or English versions of the HPLP for administration to particular individuals.

With the help of the staff of the Illinois Migrant Council Health Project, four different data-collection sites were identified in northern Illinois: a farmhouse that was a gathering place for canning factory workers, two migrant camps on a large family farm, and a "singles camp" on a turf farm and nursery. The investigator and a bilingual graduate assistant helped the volunteer participants at these sites to select the appropriate version of the research instruments by asking the first question on the acculturation scale, "What language do you prefer to speak?" To provide a larger pool for evaluation of the new translation, participants who spoke both lan- guages equally were encouraged to complete the Spanish version. The relevant language version of the demographic-acculturation questionnaire and the HPLP were administered to a total of 62 migrant farm workers. The Spanish version was completed by 36 and the English version by 26 .

The SPSSx statistical package (1986) was used to compute descriptive statistics, reliability estimates, and tests of differences between the subgroups.

\section{RESULTS}

\section{Characteristics of the Migrant Farm Workers}

Demographic characteristics of the sample are shown in Table 1. Participants' ages ranged from 18 to 61 years, with a mean of 31 years and no significant difference between the two subgroups. There was a statistically significant difference between the subgroups in gender $\left(\chi^{2}=16.10 ; P 0.001\right)$, education $(t=-2.28 ; P 0 .(126)$, and years in farm work $(t=-2.15 ; P$ 0.036). There were proportionally more men in the Spanish-speaking subgroup and more women in the English-speaking

TABLE 1. Demographic Characteristics of Mexican-American Migrant Farm Workers

\begin{tabular}{|c|c|c|c|c|c|c|}
\hline \multirow[b]{2}{*}{ Characteristic } & \multicolumn{2}{|c|}{ Total Sample } & \multicolumn{2}{|c|}{ Spanish-Speaking } & \multicolumn{2}{|c|}{ English-Speaking } \\
\hline & Number & $\%$ & Number & $\%$ & Number & $\%$ \\
\hline \multicolumn{7}{|l|}{ Sex } \\
\hline $\mathrm{M}$ & 33 & 53.2 & 27 & 75.0 & 6 & 23.1 \\
\hline $\mathrm{F}$ & 29 & 46.8 & 9 & 25.0 & 20 & 76.5 \\
\hline \multicolumn{7}{|l|}{ Marital status } \\
\hline Single & 22 & 35.5 & 14 & 38.9 & 8 & 30.8 \\
\hline Married & 32 & 51.6 & 17 & 47.2 & 15 & $57.7-7 \cdot 0.0$ \\
\hline Separated & 2 & 3.2 & 1 & 2.8 & 1 & 3.8 \\
\hline Widowed & 2 & 3.2 & 1 & 2.8 & 1 & 3.8 \\
\hline Divorced & 4 & 6.5 & 3 & 8.3 & 1 & 3.8 \\
\hline \multicolumn{7}{|l|}{ Education } \\
\hline Grade school & 22 & 35.5 & 19 & 52.8 & 3 & 11.5 \\
\hline Junior high school & 8 & 12.9 & 2 & 5.6 & 6 & 23.1 \\
\hline High school & 24 & 38.7 & 9 & 25 & 15 & $57.7-7 \cdot 0.0$ \\
\hline Trade/vocational & 5 & 8.1 & 3 & 8.3 & 2 & 7.7 \\
\hline College/university & 3 & 4.8 & 3 & 8.3 & 0 & 0.1 \\
\hline \multicolumn{7}{|l|}{ Years in farm work } \\
\hline$<12 \mathrm{mo}$ & 10 & 16.1 & 8 & 22.2 & 2 & 7.7 \\
\hline $1-4$ yrs & 15 & 24.2 & 10 & 27.8 & 5 & 19.2 \\
\hline $5-9$ yrs & 14 & 22.6 & 8 & 22.2 & 6 & 23.1 \\
\hline $10-19$ yrs & 13 & 21.0 & 6 & 16.7 & 7 & 26.1 \\
\hline$>20$ yrs & 10 & 16.1 & 4 & 11.1 & 6 & 23.1 \\
\hline Age (mean yrs) & \multicolumn{2}{|c|}{31} & \multicolumn{2}{|c|}{32.4} & \multicolumn{2}{|c|}{29.1} \\
\hline M & \multicolumn{2}{|c|}{30.2} & \multicolumn{2}{|c|}{31} & \multicolumn{2}{|c|}{26.5} \\
\hline $\mathbf{F}$ & \multicolumn{2}{|c|}{32} & \multicolumn{2}{|c|}{36.6} & \multicolumn{2}{|c|}{29.9} \\
\hline
\end{tabular}


TABLE 2. Acculturation Index of Selected Mexican-American Migrant Farm Workers

\begin{tabular}{|c|c|c|c|c|c|c|}
\hline \multirow{2}{*}{$\begin{array}{l}\text { Acculturation } \\
\text { Index Score* }\end{array}$} & \multicolumn{2}{|c|}{ Spanish-Speaking } & \multicolumn{2}{|c|}{ English-Speaking } & \multicolumn{2}{|c|}{ Total Sample } \\
\hline & Number & $\%$ & Number & $\%$ & Number & $\%$ \\
\hline 0 & 13 & 36.1 & 1 & 3.8 & 14 & 22.6 \\
\hline 1 & 19 & 52.8 & 12 & 46.2 & 31 & 50.0 \\
\hline 2 & 3 & 8.3 & 8 & 30.8 & 11 & 17.7 \\
\hline 3 & 1 & 2.8 & 4 & 15.4 & 5 & 8.1 \\
\hline 4 & 0 & 0.0 & 1 & 3.8 & 1 & 1.6 \\
\hline
\end{tabular}

$*_{0}=$ least acculturation; 4 = greatest acculturation.

TABLE 3. Perceived Health Status and Likelihood of Using Folk Medicine by Mexican-American Migrant Farm Workers

\begin{tabular}{|c|c|c|c|c|c|c|}
\hline & \multicolumn{2}{|c|}{ Spanish-Speaking } & \multicolumn{2}{|c|}{ English-Speaking } & \multicolumn{2}{|c|}{ Total Sample } \\
\hline & Number & $\%$ & Number & $\%$ & Number & $\%$ \\
\hline \multicolumn{7}{|l|}{ Perceived health } \\
\hline Excellent & 4 & 11.1 & 7 & 26.9 & 11 & 17.7 \\
\hline Good & 15 & 41.7 & 12 & 46.2 & 27 & 43.5 \\
\hline Fair & 15 & 41.7 & 7 & 26.9 & 22 & 35.5 \\
\hline Poor & 2 & 5.6 & 0 & 0 & 2 & 3.2 \\
\hline \multicolumn{7}{|c|}{ Likelihood of using folk medicine } \\
\hline Very likely & 4 & 11.1 & 2 & 7.7 & 6 & 9.7 \\
\hline Likely & 6 & 16.7 & 4 & 15.4 & 10 & 16.1 \\
\hline Unlikely & 10 & 27.8 & 9 & 34.6 & 19 & 30.6 \\
\hline Very unlikely & 16 & 44.4 & 11 & 42.3 & 27 & 43.5 \\
\hline
\end{tabular}

subgroup. The Spanish-speaking subgroup had a mean of 7.4 years of education compared to 9.4 years for the English-speaking subgroup. The English-speaking respondents had spent more years in farm work; over $70 \%$ had five years or more of experience in this work and its associated lifestyle.

Acculturation was measured by an index calculated from answers to four questions on language use, with a score of 4 indicating the highest level of acculturation and 0 indicating the lowest. As expected, the Englishspeaking workers exhibited a significantly higher level of acculturation, with $50 \%$ scoring 2 or higher on the index (Table 2). Only one worker showed complete acculturation with a score of 4 . The Spanish-speaking sample demonstrated lower levels of acculturation, with over $88 \%$ scoring 1 or less.

The Spanish-speaking workers demonstrated a significantly lower $(P<0.05)$ perception of health status in response to the question, "Considering your age, how would you describe your overall health?" (Table 3). There was no significant difference between the subgroups on likelihood of using folk medicine; nearly $26 \%$ responded that they were likely or very likely to consult a folk medicine practitioner.

\section{Measurement of Health-Promoting Lifestyle}

The participants seemed able to respond to the items on the HPLP with a few exceptions. They frequently asked questions about selected items and words. The items "Like myself" and "Touch and am touched by people I care about" often required clarification. Words and phrases that were troublesome were "artificial ingredients," "interpersonal relationships," "stretching exercises," "cholesterol level," and "environment." Once clarified, these items seemed to be understood by and relevant to the individuals completing the HPLP.

While the current sample was too small to permit comprehensive psychometric evaluation of the validity of the English and Spanish versions of the HPLP for use with this population, preliminary reliability estimates were obtained. As shown in Table 4, internal consistency of the total scale of the HPLP was adequate, with an alpha coefficient of 0.957 for the English version and 0.904 for the Spanish version. The range of alpha coefficients for the subscales was 0.558 to 0.931 for the English version. If similar values are found with larger samples, the nutrition and exercise subscales may require further development for use with English-speak- 
TABLE 4. Internal Consistency of the English and Spanish Versions of the Health-Promoting Lifestyle Profile and Its Subscales

\begin{tabular}{llll}
\hline & \multicolumn{1}{l}{ English Version } & & Spanish Version \\
\cline { 2 - 2 } Subscale & Alpha & & Alpha \\
\hline Self-actualization & 0.931 & 0.841 \\
Health responsibility & 0.786 & 0.805 \\
Exercise & 0.558 & 0.787 \\
Nutrition & 0.626 & 0.646 \\
Interpersonal & 0.903 & 0.760 \\
$\quad$ support & & \\
Stress management & 0.726 & 0.530 \\
Health-promoting & 0.957 & 0.904 \\
$\quad$ lifestyle profile & & \\
\hline
\end{tabular}

ing Hispanic populations. The range of alpha coefficients for the subscales of the Spanish version was 0.530 to 0.841 ; examination of item-total correlations identified three items that depressed the reliability of the nutrition and stress management subscales. Imprecise translation was identified as the source and this will be adjusted before the Spanish HPLP is evaluated more extensively. Conclusions based on these subscale measures in the present study must be interpreted cautiously.

\section{Health-Promoting Behaviors of the Spanish- and English-Speaking Workers}

Mean scores for the Mexican-American migrant farm workers on the HPLP are illustrated in Figure 1. The Spanish-speaking group tended to score higher than the English-speaking group on all scales except for interpersonal support. Independent $t$ tests were done to examine the significance of the difference in the HPLP total and subscale scores between the subgroups. As shown in Table 5, differences were statistically significant only for the dimensions of self-actualization, exercise, and stress management; Spanish-speaking workers reported more frequent behaviors in each of these dimensions of health-promoting lifestyle.

In further examining individual health-promoting behaviors, those that were most frequently reported by the total sample were all in the dimension of self-actualization: "look forward to the future," "am aware of what is important to me in life," and "believe that my life has purpose." The mean frequency reported for these three items was between "often" and "routinely." The nine items least often reported, as indicated by a reported frequency of "sometimes" or "never," were in four dimensions: exercise items were "participate in supervised exercise programs or activities" and "check

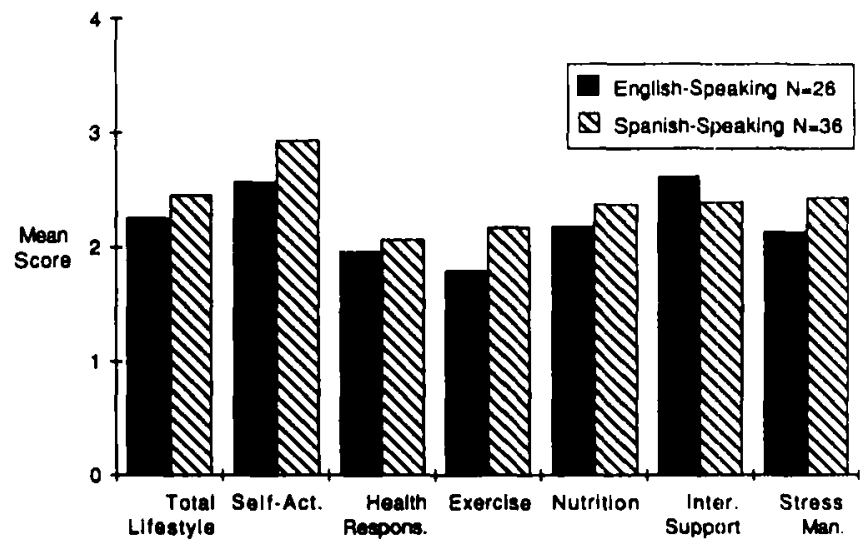

Figure 1. Health-promoting lifestyle profile scores for Englishand Spanish-speaking subgroups.

my pulse rate while exercising"; health responsibility items were "have my cholesterol checked and know the result," "attend educational programs on personal health care," "read articles or books about promoting health," and "attend educational programs on improving the environment in which we live"; the nutrition item was "choose foods without preservatives or other additives"; and stress management items were "practice relaxation or meditation for 15 to 20 minutes daily" and "use specific methods to control my stress."

Finally the total and subscale scores of both Spanishand English-speaking farm workers $(N=62)$ were compared to those of a predominantly non-Hispanic, middle-class, midwestern sample $(N=438)$ (Walker, Volkan, Sechrist, \& Pender, 1988). The total and subscale HPLP scores of the migrant farm workers appeared to be lower.

\section{DISCUSSION}

Health-promoting lifestyle initially appears to have value as a concept for further exploration among Mexican-American migrant farm workers in particular and Hispanics in general. In all instances in this study where a significant difference existed in the means between the two groups, the Spanish scores were higher, suggesting that the behaviors measured were culturally relevant and practiced even by the least-acculturated Spanishspeaking respondents. Evidence that the HPLP may be a valid measure of health-promoting lifestyle was provided by the ability of participants to respond to the items with only a few exceptions. Another observation that supported the relevance of health promotion for this group was that peer health counseling sessions spontaneously arose among participants when they had completed the HPLP. Based on the findings of this initial study, further refinements of the Spanish version of 
TABLE 5. Differences in Mean HPLP Scores Between Spanish-Speaking and English-Speaking Farm Workers

\begin{tabular}{|c|c|c|c|c|c|c|}
\hline \multirow[b]{2}{*}{ Subscale } & \multicolumn{2}{|c|}{ English } & \multicolumn{2}{|c|}{ Spanish } & \multirow[b]{2}{*}{$t$} & \multirow{2}{*}{$\begin{array}{l}\text { 2-tail } \\
P\end{array}$} \\
\hline & Mean & SD & Mean & SD & & \\
\hline Self-actualization & 2.58 & 0.65 & 2.94 & 0.49 & 2.4 & 0.02 \\
\hline Health responsibility & 1.96 & 0.52 & 2.08 & 0.54 & 0.8 & 0.42 \\
\hline Exercise & 1.81 & 0.47 & 2.18 & 0.70 & 2.51 & 0.02 \\
\hline Nutrition & 2.19 & 0.50 & 2.38 & 0.53 & 1.4 & 0.16 \\
\hline Interpersonal support & 2.60 & 0.70 & 2.41 & 0.55 & -1.2 & 0.24 \\
\hline Stress management & 2.14 & 0.48 & 2.4 & 0.43 & 2.4 & 0.02 \\
\hline Health-promoting lifestyle & 2.26 & 0.49 & 2.5 & 0.37 & 1.7 & 0.09 \\
\hline
\end{tabular}

the HPLP and psychometric evaluation of its reliability and validity among a larger and more diverse Hispanic population will be undertaken.

Lifestyle practices among this group of MexicanAmerican workers appeared to be strong in the areas of interpersonal support and self-actualization, with no infrequently reported health behavior items from either of these subscales. The interpersonal support subscale measures relationships involving a sense of intimacy and closeness (Walker et al., 1988). It is not surprising that this is an area of strength for this group in light of the widely recognized cultural importance MexicanAmericans ascribe to the family (Friedman, 1986). The family's purpose is to provide support for its members, a function that is apparently viable even for members who are geographically distant from the family core. The sense of belonging and the assurance of emotional support are internalized within each person, enabling migrant family members to contribute financially to the rest of the family. The culture itself seems to enhance the health-promoting strength of interpersonal support.

On the other hand, strength in the area of self-actualization was a surprising finding in view of the powerlessness and entrapment inherent in migrant farm work. This subscale measures the individual's sense of purpose, personal development, self-awareness, and satisfaction (Walker et al., 1988). The authors can only speculate as to the role of culture in promoting selfactualization. If Mexican-American individuals see themselves as members of the family and community first, and as individuals second (Friedman, 1986), the accomplishment of family or community goals may be self-actualizing for each one. Further study is required to determine the extent and significance of self-actualizing health behaviors in this population.

Analysis of the infrequently reported health behavior items suggests several needs. The issue of access to health resources is implicit in many items; cholesterol screening, supervised exercise programs, and educational resources such as books or programs are seldom available to low-income families living in a rural environment. Similarly, migrant farm workers may not have knowledge of specific health-promoting behaviors such as practicing relaxation techniques and checking their pulse rates. Language may form a formidable barrier for Spanish-speaking persons since nutrient levels on packaged foods and health education information are generally in English.

A possible explanation for the significantly lower score of the English-speaking workers on three subscales of the HPLP is that they may have had different reading skills. Workers who were fluent in spoken English may still have had difficulty reading the language and might therefore have answered "never" to health behaviors they did not comprehend. Analysis of the demographics of the Spanish-speaking group may offer other insights. The Spanish-speaking sample was composed primarily of men working at a turf farm, and therefore characteristics of their lifestyle would have had a strong influence on their response on the HPLP.

A singles camp is usually composed of men traveling alone without their families. Several of the men had questioned the researcher about their marital status as to whether it meant here or at home in Mexico; they then responded "married." Their significantly higher self-actualization score may in part be explained by anecdotes. The men stated that they earn a good income by working many hours from dawn to dusk with very few days off. They explained that their wives and children were in Mexico and that they worked hard in order to send money home. It is possible that these men selfactualize by daily achieving their goal to maintain financial stability for their families in Mexico. The higher exercise score may relate to the physically demanding nature of the work on a sod farm/nursery, or may reflect the fact that many of these men participated in an evening soccer league.

The lower health-promoting lifestyle scores of these farm workers compared with middle-class midwesterners is not surprising in view of their lack of access to 
health-promotion resources and information, and their mobile, hard-working lifestyle necessary for survival. Further development of the Spanish version of the instrument would allow investigation of the subscales to identify subcategories of health-promoting lifestyle that may be strengths for the Mexican-Americans.

The range of acculturation scores within the Englishand Spanish-speaking groups illustrates the diversity of this population. Each subgroup contained persons with varying degrees of acculturation. As a whole, they retained aspects of their mother language and culture; only one person had a score that illustrated full acculturation.

The workers' perceived health status compared very closely to that of workers in a Wisconsin study (Slesinger, 1981) and was even lower than the perceived health status of Mexican-Americans in Alameda County (Roberts \& Lee, 1980). Since perception of health is a subjective measure that depends on one's definition of health, it might be more valid to identify this in Mexican-American migrant farm workers, rather than compare this group to other cultural groups who may have a different concept of health.

\section{NURSING IMPLICATIONS}

Although further development of the Spanish version of the HPLP is indicated before conclusions are drawn on the basis of subscale results, it appears that the behaviors and attitudes measured are practiced by at least some of the migrant farm workers. Having identified strengths among a particular group, the challenge for nurses is to capitalize on and enhance those strengths in efforts to promote health. For example, strong interpersonal support can be used as both rationale and encouragement for engaging in health-promoting activities: "Your family cares about you a great deal; they want you to be as healthy as you can be and they will help you achieve that goal."

While further research is necessary to define specific areas that must be strengthened, analysis of the healthpromoting behaviors practiced least frequently suggests the following potential needs for Mexican-American migrant farm workers: (1) increased access to health resources, (2) increased knowledge of health-promoting behaviors, and (3) decreased language barriers. These are familiar challenges for nurses working in migrant health since they also relate to illness prevention and health maintenance.

Access to health resources can be increased by bringing the assessment tools and educational programs to the migrant camp or work setting whenever possible. For example, the use of a portable cholesterol-screening instrument in community health practice may already be having an impact on farm workers' knowledge of their cholesterol level (personal communication, M. J. Kulka, December 1988). Organizing team sports or exercise clubs is a way to promote fitness within a community setting. Access in this case may involve simply acquiring a soccer ball and permission to use space for a playing field.

Knowledge of health-promoting behaviors is an important step toward lifestyle change. Teaching such behaviors and lifestyle-modification skills could take many forms with individuals, families, or groups. Building on current knowledge and healthful practices can improve receptivity to and retention of new information. For example, affirming the traditional meal of beans and tortillas is a starting point for nutrition education. If beans are refried in vegetable oil instead of lard, this meal is quite healthful. As one Hispanic activist stated, "We've been eating beans and tortillas for generations because that's all we had to eat ... and now they tell us it's good for us!" When nurses begin by reinforcing positive behaviors, their clients are very likely to be open to suggestions for improvements in other areas. As various interventions for lifestyle change are implemented with this population, research to evaluate their effectiveness will be necessary.

To decrease language barriers, health information in Spanish and bilingual community health workers are essential. Consumer English classes could be developed in which Spanish-speaking persons learn to read ingredient and nutrient labels on packaged foods. Walletsized cards with lists of ingredients to avoid and which to include in a healthy diet would be helpful for Englishas well as Spanish-speaking consumers engaging in comparative shopping. As the Hispanic population in the United States grows, support of nutritional labeling in Spanish is an appropriate role for the community health nurse. Although many organizations do provide health information in the Spanish language, it is often a matter of chance that these become available at the time they are needed. In this age of information technology, it would be timely to create a national computerized information system of Spanish-language health literature, audiovisual aids, and health-assessment tools.

This study suggests several areas for future research; develop reliable and valid research instruments in the Spanish language, investigate self-actualization in the Mexican-American migrant farm worker culture, identify the influence of culture on health attitudes and behaviors in each of the six dimensions of health-promoting lifestyle, and evaluate specific interventions for their effectiveness in producing positive outcomes for these workers and their families. Further research in the area of health promotion with all cultural groups and 
socioeconomic levels of society will contribute to achievement of health for all by the year 2000 .

\section{ACKNOWLEDGMENTS}

The authors gratefully acknowledge the consultation of Susan Noble Walker, Ed.D., R.N.; the assistance of Julie Robertson M.S.N., R.N. in reviewing the manuscript; and the help of Susan Bauer, M.P.H., Mary Jule Kulka, M.S., R.N., and Carlos Rodriguez of the Illinois Migrant Council Health Project in planning this study and translating the instrument.

\section{REFERENCES}

Berkanovic, E. (1980). The effect of inadequate language translation on Hispanics' responses to health surveys. American Journal of Public Health, 70, 1273-1276.

Deyo, R. (1984). Pitfalls in measuring the health status of Mexican Americans: Comparative validity of the English and Spanish sickness impact profile. American Journal of Public Health, 74, 569-573.

Deyo, R., Diehl, A., Hazuda, H., \& Stern, M. (1985). A simple language-based acculturation scale for Mexican Americans: Validation and application to health care research. American Journal of Public Health, 75, 51-55.

Friedman, M. M. (1986). Family nursing: Theory and assessment (2nd ed.). Norwalk, CT: Appleton-Century-Crofts.

Gottlieb, N., \& Green, L. (1987). Ethnicity and lifestyle health risk: Some possible mechanisms. American Journal of Health Promotion, 2, 37-51.

Marks, G., Solis, J., Richardson, J., Collins, L., Lourdes, B., \& Hisserich, C. (1987). Health behavior of elderly Hispanic women: Does cultural assimilation make a difference? American Journal of Public Health, 77, 1315-1319.
Pender, N. (1987). Health promotion in nursing practice. Norwalk, CT: Appleton-Century-Crofts.

Roberts, R., \& Lee, E. (1980). The health of Mexican Americans: Evidence from the human population laboratory studies. American Journal of Public Health, 70, 375-384.

Slesinger, D. (1981). Medical utilization patterns of Hispanic migrant farmworkers in Wisconsin. Public Health Reports, 96, 255-263.

Slesinger, D. (1988). Health status of Wisconsin's migrant agricultural workers. As you sow ... Social issues in agriculture, 19, 1-2.

Smith, K. (1986). The hazards of migrant farm work: An overview for rural public health nurses. Public Health Nursing. 3, 48-56.

SPSS Inc. (1986). SPSSx user's guide (2nd ed.). New York: McGraw-Hill.

Stern, M., Pugh, J., Gaskill, S., \& Hazuda, H. (1982). Knowledge, attitudes, and behavior related to obesity and dieting in Mexican Americans and Anglos: The San Antonio heart study. American Journal of Epidemiology, 115, 917-928.

Walker, S., Sechrist, K., \& Pender, N. (1987). The healthpromoting lifestyle profile: Development and psychometric characteristics. Nursing Research, 36, 76-81.

Walker, S., Volkan, K., Sechrist, K., \& Pender, N. (1988). Health-promoting life styles of older adults: Comparisons with young and middle-aged adults, correlates and patterns. Advances in Nursing Science, 11, 76-90.

Wilk, V. (1986). The occupational health of migrant and seasonal farmworkers in the United States. Washington, DC: Farmworker Justice Fund.

World Health Organization. (1982). Seventh general programme of work covering the period 1984-89 (Health for All series, no. 8). Geneva: Author. 